\title{
MITTELLATEINISCHES WÖRTERBUCH
}

BIS ZUM AUSGEHBNDEN 13. JAHRHUNDERT

\author{
In Gemeinschaft mit \\ den Akademien der Wissenschaften zu Göttingen, Heidelberg, Leipzig, Mainz, Wien \\ und der Schweizerischen Geisteswissenschaftlichen Gesellschaft \\ herausgegeben \\ von der \\ Bayerischen Akademie der Wissenschaften \\ und der
}

Deutschen Akademie der Wissenschaften zu Berlin

I. Band. Lieferung 9

authentisatus - beneficium

AKADEMIE-VERLAG GMBH, BERLIN

1966 
Die Arbeiten in München werden von der Deutschen Forschungsgemeinschaft gefördert.

Vorstand der Kommission:

Paul Lehmann $\dagger$, München, Johannes Stroux†, Berlin, Bernhard Bischofr, München

Redaktion:

Otto Prinz, München, unter Mitarbeit von Johannes SCHNErDER, Berlin

Anschriften:

MrTTEILATERNISCHES WÖRTERBUCH

Münchner Arbeitsstelle: München 22, Marstallplatz 8 (Residenz)

Berliner Arbeitsstelle: Berlin W 8, Otto-Nuschke-Straße 22-23

Lizenz-Ausgabe für die DDR, die UdSSR, Polen, die Tschechoslowakei, Ungarn, Rumänien, Bulgarien, Albanien und Jugoslawien, alle Länder Asiens mit Ausnahme von Japan, für Ägypten, den Sudan, Nigeria und Ghana.

(C) Alle Verlagsrechte bei der C. H. Beck'schen Verlagsbuchhandlung (Oscar Beck) München. 1966

Druck der C.H. Beck'schen Buchdruckerei, Nördlingen

Printed in Germany 